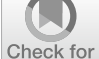

Check for

updates

Cite as

Nano-Micro Lett.

(2021) $13: 145$

Received: 17 March 2021

Accepted: 11 May 2021

Published online: 19 June 2021

(C) The Author(s) 2021

\section{Targeted Micellar Phthalocyanine for Lymph Node Metastasis Homing and Photothermal Therapy in an Orthotopic Colorectal Tumor Model}

\author{
Hai-Yi Feng ${ }^{1,2}$, Yihang Yuan ${ }^{1}$, Yunpeng Zhang ${ }^{2}$, Hai-Jun Liu ${ }^{1}$, Xiao Dong ${ }^{1}$, \\ Si-Cong Yang ${ }^{1}$, Xue-Liang Liu ${ }^{1}$, Xing Lai ${ }^{1}$, Mao-Hua Zhu ${ }^{1}$, Jue Wang ${ }^{2}$, Qin Lu ${ }^{1}$, \\ Quanjun Lin ${ }^{2}$, Hong-Zhuan Chen ${ }^{3}$, Jonathan F. Lovell ${ }^{4}$, Peng Sun ${ }^{2}{ }^{凶}$, Chao Fang ${ }^{1} \bowtie$
}

\title{
HIGHLIGHTS
}

- Small-sized trastuzumab-targeted micelles (T-MP) were engineered using a surfactant-stripping approach that yielded concentrated phthalocyanines with strong near infrared absorption.

- T-MP accumulated more in the lymph node (LN) metastases of orthotopic colorectal cancer compared to the micelles conjugated with control IgG.

- Following surgical resection of the primary tumor, minimally invasive photothermal treatment of the metastatic LN with T-MP, but not the control micelles, extended mouse survival.

ABSTRACT Tumor lymph node (LN) metastasis seriously affects the treatment prognosis. Studies have shown that nanoparticles with size of sub-50 nm can directly penetrate into LN metastases after intravenous administration. Here, we speculate through introducing targeting capacity, the nanoparticle accumulation in LN metastases would be further enhanced for improved local treatment such as photothermal therapy. Trastuzumabtargeted micelles $(<50 \mathrm{~nm})$ were formulated using a unique surfactantstripping approach that yielded concentrated phthalocyanines with strong near-infrared absorption. Targeted micellar phthalocyanine (T-MP) was

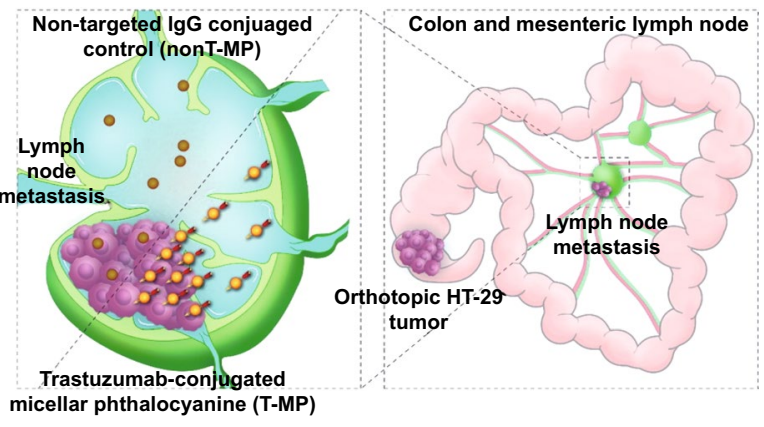
an effective photothermal transducer and ablated HT-29 cells in vitro. A HER2-expressing colorectal cancer cell line (HT-29) was used to establish an orthotopic mouse model that developed metastatic disease in mesenteric sentinel LN. T-MP accumulated more in the LN metastases compared to the micelles conjugated with control IgG. Following surgical resection of the primary tumor, minimally invasive photothermal treatment of the metastatic LN with T-MP, but not the control micelles, extended mouse survival. Our findings demonstrate for the first time that targeted small-sized nanoparticles have potential to enable superior paradigms for dealing with LN metastases.

KEYWORDS Lymph node metastasis; Photothermal therapy; Trastuzumab; Phthalocyanine; Micelles

Hai-Yi Feng and Yihang Yuan contributed equally to this work.

$\triangle$ Peng Sun, sp2082@shtrhospital.com; Chao Fang, fangchao32@sjtu.edu.cn

1 Hongqiao International Institute of Medicine, Tongren Hospital and State Key Laboratory of Oncogenes and Related Genes, Department of Pharmacology and Chemical Biology, Shanghai Jiao Tong University School of Medicine (SJTU-SM), Shanghai 200025, People's Republic of China

2 Department of General Surgery, Tongren Hospital, SJTU-SM, Shanghai 200336, People's Republic of China

3 Institute of Interdisciplinary Integrative Biomedical Research, Shuguang Hospital, Shanghai University of Traditional Chinese Medicine, Shanghai 201203, People's Republic of China

4 Department of Biomedical Engineering, University at Buffalo, State University of New York, Buffalo, NY 14260, USA 


\section{Introduction}

Solid tumor progression is usually accompanied by lymphatic metastasis, which seriously affects the disease prognosis $[1,2]$. Lymphadenectomy is the dominant method for treating lymphatic metastasis in cancer clinic [3]. However, this surgical operation is mainly based on surgeons' clinical practice experience, such as the D2 lymphadenectomy for gastric cancer management [4], which does not accurately distinguish between metastatic and healthy lymph nodes (LNs). Due to the lack of reliable method for metastasis identification and lymphatic imaging, precise clearance of the metastatic LNs still remains a big challenge. Incomplete lymphadenectomy may finally end up with disease recurrence, and excessive resection would lead to a higher incidence of postoperative complications [3, 4]. Near-infrared (NIR) fluorescent imaging after peritumoral injection of indocyanine green (ICG) offers an effective method to visualize the lymphatic anatomy for surgeons [5]. However, the local injection of the imaging agent is restricted due to the disadvantage of "dye spillage" which makes high background signals [6].

Systemic chemotherapy for the LN metastasis usually requires high doses and inevitably increases the risk of dose-limiting toxicities [7]. Thus, eradicating the tumor cells in the LN metastases by targeted drug delivery to the metastatic LNs is a more attractive solution [8]. However, most of the preclinical efforts on tumor metastatic LN targeted drug delivery utilize the subcutaneous tumor model, such as the mostly used xenograft model in the mouse footpad $[9,10]$. Nanoparticles are administered through the interstitial space next to the tumor to target locally metastatic LNs. Generally, such tumor models and local delivery methods are lack of clinical relevance. In fact, in contrast to lots of reports aiming to improve nanoparticle penetration in tumor primary site [11], relatively few efforts have been made for LN metastasis penetration and distribution.

Recently, systemic administration of nanoparticles has been explored to target LN metastasis. Through enhancing the penetration in tumor tissues by $\mathrm{pH}$-responsive size reduction from 100 to $5 \mathrm{~nm}$ at the tumor site, nanoparticles were allowed to enter into the LNs through tumor-draining lymphatics $[6,12]$. Lymphatic vessels of solid tumors are poorly developed, which is a key pathophysiological basis of the enhanced permeation and retention (EPR) effect
[13-15], and in most clinical cancer settings, chemotherapy is performed after the primary tumor is resected. Therefore, the application of this method after tumor resection might be restricted. Compared with large-sized particle, intravenously administered nanocarriers with smaller dimension $(<50 \mathrm{~nm})$ can specifically extravasate from the blood vessels in LN metastases and accumulate, while their distribution in healthy LNs was significantly low [7]. This size-controlled targeting to the LN metastasis does not rely on the existence of the primary tumor [7] and thus holds potential for wider application.

It has been shown that tumor cell-targeted nanocarriers can accumulate more in the primary lesion of solid tumors compared the non-targeted counterparts and therefore confer enhanced therapeutic outcome [16]. We thus hypothesize that through endowing nanocarriers with targeting property for tumor cells, furtherly enhanced nanoparticle accumulation in LN metastasis would be achieved, which may provide greater benefits for the local treatment (such as photothermal therapy) $[17,18]$ of LN metastasis.

HER2 (human epidermal growth factor receptor 2)-targeted micellular phthalocyanine with size below $50 \mathrm{~nm}$ was developed as illustrated in Scheme 1a. The near-infrared (NIR) chromophore VBPc (Vanadyl 3,10,17,24-tetratert-butyl-1,8,15,22-tetrakis(dimethylamino)-29H,31Hphthalocyanine, a photothermal and photoacoustic inducer) was loaded into carboxylated F127 micelles using the emerging surfactant-stripping strategy, taking advantage of the temperature-dependent CMC (critical micelle concentration) of F127 that enables removal of free and loosely bound surfactant using membrane filtration at $4{ }^{\circ} \mathrm{C}$ [19-21]. This generates concentrated VBPc micelles containing minimal solubilizing excipient and intense NIR absorption to enable both photoacoustic imaging and photothermal properties. Next, trastuzumab, a human antiHER 2 antibody, was conjugated to the micelle surface through EDS/NHS chemistry to generate trastuzumab-conjugated targeted micellar phthalocyanine (T-MP). T-MP would enable targeting to HER 2 expressed on the surface of tumor cells, such as HT-29 colorectal cancer cells [22, 23]. An orthotopic HT-29 colorectal tumor model was established [24], and metastasis to the mesenteric sentinel LN was observed. It was postulated that after intravenous (i.v.) injection, the small-sized T-MP can accumulate more in the $\mathrm{LN}$ metastasis compared to the non-targeted isotype IgG-conjugated controls (nonT-MP) (Scheme 1b), 
which may lead to an improved photothermal therapy for the LN metastases. To our knowledge, this work is the first report demonstrating that through targeting modification, i.v. injected small-sized nanoparticles can achieve more accumulation in LN metastases for improved therapeutic outcomes (photothermal treatment in this study), which are also superior to the traditional lymphadenectomy that usually incurred surgical trauma and postoperative complications. The multifunctional properties of T-MP were characterized, and their potency in overcoming LN metastasis in the metastatic orthotopic HT-29 colorectal tumor model was investigated.

\section{Experimental Section}

\subsection{Materials, Cell Culture, and Animals}

Pluronic F127 and Vanadyl 3,10,17,24-tetra-tert-butyl1,8,15,22-tetrakis(dimethylamino)-29H,31H-phthalocyanine (VBPc) were supplied by Sigma-Aldrich (Shanghai, China). 1-ethyl-3-(3-dimethylaminopropyl) carbodiimide (EDC) and $\mathrm{N}$-hydroxysuccinimide (NHS) were obtained from Aladdin
Chemistry Company (Shanghai, China). Trastuzumab (Herceptin) was obtained from Tongren Hospital (Shanghai, China). Rabbit anti-HER2 antibody and FITC/HRP goat anti-rabbit antibody were obtained from Abcam (Shanghai, China). Cy5.5 NHS ester was purchased from Lumiprobe (Hunt Valley, Maryland). iFluor 594 NHS ester was obtained from AAT Bioquest (Sunnyvale, CA). McCoy's 5A Medium, fetal bovine serum (FBS), Dulbecco's phosphate-buffered saline (DPBS), penicillin, and streptomycin were purchased from Thermo Fisher Scientific. Double distilled water was purified using a Millipore simplicity system (Millipore, Bedford, MA). All other chemicals were of analytical grade and used without further purification.

HT-29 human colorectal adenocarcinoma cell line was obtained from the ATCC (Manassas, VA). HT-29 cells transfected with luciferase (HT-29-luc) were constructed by Shanghai Model Organisms Center (Shanghai, China). Cells were cultured in McCoy's 5A medium with $10 \%$ FBS, $10^{5} \mathrm{U}$ $\mathrm{L}^{-1}$ penicillin, and $100 \mathrm{mg} \mathrm{L}^{-1}$ streptomycin.

Female BALB/c nude mice (4-5 weeks old) were provided by Shanghai Laboratory Animal Center (Chinese Academy of Sciences, Shanghai, China). The animal

(a)

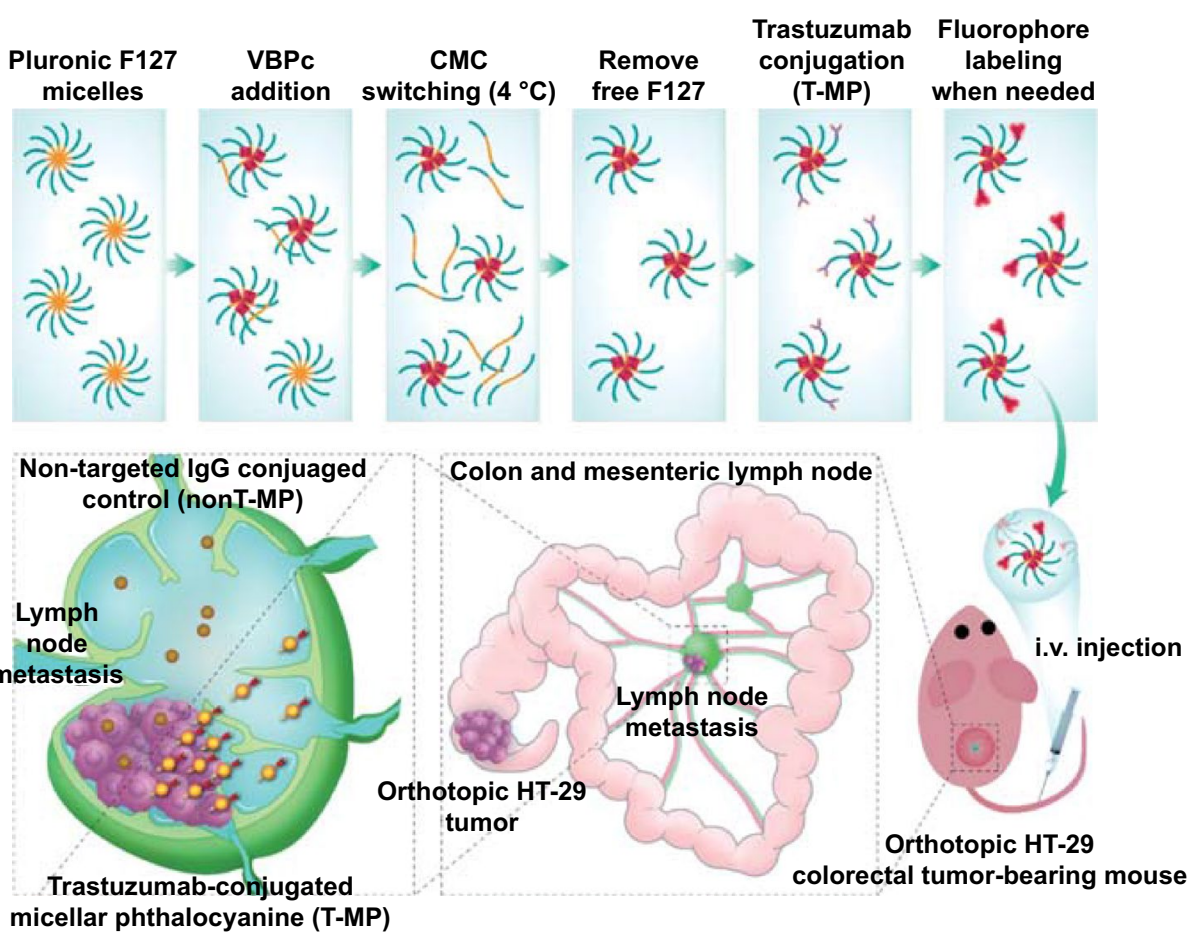

Scheme 1 Schematic illustration of the engineering of surfactant-stripped targeted micellar phthalocyanine (T-MP) (a) and improved LN metastasis targeting using this approach $(\mathbf{b})$ 
experiment designed in this study was approved by the ethical committee of SJTU-SM.

\subsection{Preparation and Characterization of Targeted Micellar Phthalocyanine (T-MP)}

The micellular phthalocyanine was formed through selfassembly of Pluronic F127 and VBPc [19]. $2 \mathrm{mg}$ VBPc in $1 \mathrm{~mL}$ dichloromethane was added dropwise to $10 \mathrm{~mL} 10 \%$ (w/v) Pluronic F127 (containing 15\% carboxylated polymer prepared as described) [25]. The solution was stirred at room temperature until the dichloromethane was evaporated. After centrifugation at $5000 \mathrm{~g}$ for $5 \mathrm{~min}$, the supernatant was purified through CMC switching. For this procedure, ultrafiltration using Amicon Ultra-15 centrifugal filtration device (100 KD MWCO) at $4{ }^{\circ} \mathrm{C}$ was performed until $200 \mu \mathrm{L}$ solution was retained. Distilled water was then added back into the filtration device, and the washing procedure was repeated twice.

Antibody modification is a common route to confer targeting property of the nanocarriers [26]. For trastuzumab conjugation, the antibody was mixed with the micellar VPBc at 1:3 molar ratio of trastuzumab to carboxyl groups of micelles in the presence of EDC and NHS. The solution was then stirred for $12 \mathrm{~h}$ at $4{ }^{\circ} \mathrm{C}$. The resulting targeted micellar phthalocyanine (T-MP) was purified by ultrafiltration to remove excess EDC and NHS.

The VBPc fluorescence was quenched due to the aggregation in the micellar core. For the fluorescence labeling of T-MP, Cy5.5 or iFluor 594 NHS ester was incubated with T-MP in dark for $1 \mathrm{~h}$ (1:5 molar ratio of trastuzumab to dye). Excess dyes were removed by ultrafiltration at $5000 \mathrm{~g}$ for 3 times.

The morphology of the resulting T-MP was observed using transmission electron microscopy (TEM, FEI Talos F200X). The hydrodynamic size and zeta potential of nanoparticles were determined through dynamic light scattering (DLS) method and measured by ZetaSizer Nano ZS instrument (Malvern, Worcestershire, the UK). Vis-NIR absorbance of free VBPc in dichloromethane and unmodified MP in water was examined on a spectrofluorophotometer (Thermo Scientific Varioskan Flash). The carboxylation of F127 was examined using Fourier transform infrared spectroscopy (FTIR). The antibody conjugation was identified using FTIR spectroscopy and X-ray photoelectron spectroscopy (XPS) assay. The conjugated trastuzumab and the linked Cy5.5 fluorophore on the micelles were also identified by non-reducing sodium dodecyl sulfate-polyacrylamide electrophoresis (SDS-PAGE) and NIRF imaging using the IVIS Spectrum CT imaging system (PerkinElmer). For VBPc loading determination, the micelles were lyophilized, weighted, and then destroyed in DMSO. The dissolved VBPc was measured by UV absorption at $800 \mathrm{~nm}$ for the estimation of drug loading.

\subsection{Photothermal Heating and Photoacoustic Imaging in Vitro}

T-MP with different concentrations of VBPc $(10,20,50$, and $\left.100 \mu \mathrm{g} \mathrm{mL}^{-1}\right)$ in 96 -well plates was irradiated by $808 \mathrm{~nm}$ laser $\left(2 \mathrm{~W} \mathrm{~cm}^{-2}\right)$ for $5 \mathrm{~min}$. Temperature increases were monitored using the Pt100 temperature probe (Testo, Shanghai, China), and the photothermal images were recorded using thermal imaging camera (Testo 890). The laser power $\left(1,1.5\right.$, and $\left.2.5 \mathrm{~W} \mathrm{~cm}^{-2}\right)$-dependent temperature increase was evaluated with $20 \mu \mathrm{g} \mathrm{mL}^{-1} \mathrm{VBPc}$-contained T-MP. The photothermal stability was also examined using $20 \mu \mathrm{g} \mathrm{mL}^{-1}$ of T-MP $(200 \mu \mathrm{L})$ under $2 \mathrm{~W} \mathrm{~cm}^{-2}$ laser irradiation for three cycles. Photothermal conversion efficiency was calculated according to methods described in the literature [27-29].

For photoacoustic (PA) imaging evaluation, T-MP suspensions were immobilized in the polyurethane tubings $(0.015$ ID $\times 0.033$ OD). A Vevo LAZR-X multimodal imaging system (Fujifilm VisualSonics) was used to acquire the PA images at an excitation of $770 \mathrm{~nm}$. Different concentrations $\left(2,5,20,50\right.$, and $\left.100 \mu \mathrm{g} \mathrm{mL}{ }^{-1} \mathrm{VBPc}\right)$ of T-MP were tested. The quantified PA signal intensity within region of interest (ROI) of each image was analyzed using the LAZR software (Fujifilm VisualSonics).

\subsection{Cellular Uptake and Photothermal-Induced Cytotoxicity}

HT-29 cells were cultured in $96-$ well plate $\left(1 \times 10^{4}\right.$ cells per well). When the cells reached about $80 \%$ confluence, the culture medium was replaced with $200 \mu \mathrm{L}$ iFluor 594-labeled micelles (iFluor $5940.1 \mu \mathrm{g} \mathrm{mL}^{-1}$ ) for 1 and $4 \mathrm{~h}$, respectively. Then, the cells were fixed with a $4 \%$ paraformaldehyde solution for $15 \mathrm{~min}$, and the cell nuclei were stained with $100 \mathrm{ng} \mathrm{mL}^{-1}$ Hoechst 33,342 for $8 \mathrm{~min}$. The cellular uptake 
was assayed by quantifying the intracellular fluorescence intensity on a Thermo Scientific ArrayScan XTI High Content Analysis Reader (Thermo Fisher Scientific Cellomics, the USA). In another assay, the cells were seeded on the coverslip in 24 -well plate $\left(1 \times 10^{5}\right.$ per well). The images of cellular uptake were obtained using confocal laser scanning microscopy (CLSM) (Leica TCS SP8, Germany; iFluor 594: $\left.E_{\mathrm{x}} 563 \mathrm{~nm}, E_{\mathrm{m}} 604 \mathrm{~nm}\right)$.

For the evaluation of photothermal-induced cytotoxicity, HT-29 cells were seeded in 96-well plates at the density of $1 \times 10^{4}$ cells per well. After $24 \mathrm{~h}$ culture, the cells were incubated with $20 \mu \mathrm{g} \mathrm{mL}^{-1} \mathrm{VBPc}-$ contained T-MP for $4 \mathrm{~h}$. Then, the cells were incubated in fresh medium and irradiated with $808 \mathrm{~nm}$ laser $\left(2 \mathrm{~W} \mathrm{~cm}^{-2}\right)$ for $20 \mathrm{~min}$. After $24 \mathrm{~h}$, the cell viability was assayed using Cell Counting Kit- 8 according to the manufacturer's instructions or calcein-AM/PI dualstaining test as we previously described [30]. Laser only, nonT-MP alone, or T-MP alone were included as controls.

\subsection{Orthotopic Colorectal Cancer Model and LN and Organ Metastasis Profile}

The orthotopic colorectal cancer model was established as described in our previous report [24]. Briefly, female $\mathrm{BALB} / \mathrm{c}$ nude mice were anesthetized with ketamine and xylazine. A $2-3 \mathrm{~cm}$ abdominal midline incision was made to expose the mouse cecum. Then, $2 \times 10^{6}$ HT-29-luc cells suspended in $50 \mu \mathrm{L}$ culture medium were orthotopically inoculated into the subserosa layer of the cecum wall using 30 gauge needle (Hamilton, Reno, NV). The injection site was pressed with a cotton swab for 1-2 min to prevent cell effusion. The cecum was placed back into the peritoneal cavity, and the abdominal wall and skin were sutured with 5-0 suture, respectively.

The tumor formation and growth with the time were monitored using the IVIS Spectrum CT imaging system (PerkinElmer). Typically, the mice were intraperitoneally injected with d-luciferin (150 $\mathrm{mg} \mathrm{kg}^{-1}$, J\&K Chemical, Shanghai, China). After $8 \mathrm{~min}$, the mice were anesthetized and imaged under the imaging system to examine the bioluminescence.

For $\mathrm{LN}$ and organ metastasis profile monitoring, mice at different time points (15 d, 22 d, 29 d, 36 d) after tumor cell inoculation were sacrificed. The LNs at three anatomical positions in the peritoneal cavity (pancreaticoduodenal, mesenteric, and lumbar LNs) and major organs (heart, liver, spleen, lung, and kidney) were excised for ex vivo bioluminescence imaging to examine the metastasis.

\subsection{Metastatic Mesenteric Sentinel LN Targeting and Biodistribution of T-MP}

Cy5.5-labeled T-MP (Cy5.5 $0.1 \mathrm{mg} \mathrm{kg}^{-1}$ ) was i.v. injected to HT-29-luc orthotopic tumor-bearing mice. After $2,4,8$, and $24 \mathrm{~h}$, respectively, the mice $(n=3$ per group) were sacrificed, and the mesenteric sentinel LN was excised for fluorescence imaging assay using the IVIS Spectrum CT imaging system ( $E_{\mathrm{x}} 685 \mathrm{~nm}, E_{\mathrm{m}}$ $710 \mathrm{~nm}$ ). The Cy5.5-labeled nonT-MP was included as control. The fluorescence intensities in the LNs were quantified and compared. LN targeting was also identified using PA imaging. The tumor-bearing mice $(n=3$ per group) were i.v. injected with the micelles (VBPc $5 \mathrm{mg} \mathrm{kg}^{-1}$ ). After $8 \mathrm{~h}$, the mesenteric sentinel LNs were excised for PA imaging, and the PA signal intensities of T-MP and nonT-MP in the LNs were recorded and compared. To examine the micro-distribution of the micelles, the LNs were processed for frozen section. The metastases (green; $E_{\mathrm{x}} 488 \mathrm{~nm}, E_{\mathrm{m}} 520 \mathrm{~nm}$ ) were stained using rabbit anti-HER2 antibody and FITC goat anti-rabbit secondary antibody. The image was obtained using Zeiss Axio Scan.Z1 slide scanner. The distribution of Cy5.5 labeled micelles (red; $E_{\mathrm{x}} 633 \mathrm{~nm}, E_{\mathrm{x}} 710 \mathrm{~nm}$ ) in the metastasis and non-metastasis region was observed and analyzed using ZEN 2.3 slidescan software.

For biodistribution study, mice at $8 \mathrm{~h}$ after injected with Cy5.5-labeled micelles were sacrificed. The tumor and major organs (heart, liver, spleen, lung, and kidney) were excised for fluorescence imaging assay in the IVIS Spectrum CT imaging system.

For VBPc assay, $50 \mathrm{mg}$ of tissue or the weighted mesenteric sentinel LNs were homogenized with $3 \mathrm{~mL} 2 \%$ SDS. Then, $500 \mu \mathrm{L}$ of the homogenate was added with $500 \mu \mathrm{L}$ of methanol and chloroform (v/v 1:2) for 1 min vortex. The mixture was then centrifuged $(5000 \mathrm{~g}, 20 \mathrm{~min})$, and the supernatant was taken to detect the absorption at $808 \mathrm{~nm}$ for VBPc determination. 


\subsection{Photothermal Therapy of Metastatic Mesenteric Sentinel LNs and Antitumor Evaluation}

Female BALB/c nude mice $(n=5)$ bearing orthotopic colorectal tumor were divided into six groups as following: (1) Untreated, (2) Tumor resection, (3) nonT-MP, (4) T-MP, (5) nonT-MP + Laser, and (6) T-MP + Laser. For group (3), (4), (5), and (6), tumor resection was also performed. The micelles with VBPc $5 \mathrm{mg} \mathrm{kg}^{-1}$ for each mouse were i.v. injected to the mice when involved. $8 \mathrm{~h}$ after injection, the orthotopic tumor was resected, and the mesenteric sentinel LN was irradiated using $808 \mathrm{~nm}$ laser $\left(2 \mathrm{~W} \mathrm{~cm}^{-2}, 10 \mathrm{~min}\right)$. An infrared thermal camera (Testo 890) was used to monitor the temperature change of the LN during the laser irradiation. Then, the abdominal wall and skin were sutured. To examine the effect on the LN microstructure, the LNs were excised for ultrathin section making and observed under TEM [31].

The tumor growth and recurrence were monitored by bioluminescence imaging every 7-8 days. The mouse survival time and body weight were monitored every other day post various treatments. Increase in life span (ILS) was calculated using the formula: $\%$ ILS $=(T / C-1) \times 100 \%$ [32]. $T$ and $C$ were the median survival of the tumor-bearing mice in the treated and untreated groups, respectively.

\subsection{Histopathological and Immunohistochemical Assay}

To identify the LN metastases, the mesenteric sentinel LNs of the mice were excised and processed for hematoxylin and eosin (H\&E) staining and pathological examination. The HER2-positive metastases in LNs and the specimens of human colorectal cancer were also demonstrated through rabbit antiHER2 antibody immunohistochemical staining.

To investigate the toxicity of micelles on the organs, $24 \mathrm{~h}$ after i.v. injection of micelles (VBPc $5 \mathrm{mg} \mathrm{kg}^{-1}, n=3$ ), major organs (heart, liver, spleen, lung, and kidney) of the tumorbearing mice were excised and processed for $\mathrm{H} \& \mathrm{E}$ staining and pathological examination under photomicroscope (Nikon Eclipse E200).

\subsection{Blood Analysis}

Healthy female BALB/c nude mice were i.v. injected with micelles (VBPc $5 \mathrm{mg} \mathrm{kg}^{-1}$ ). After 24, 96, and $169 \mathrm{~h}$, respectively, retro-orbital blood was collected for serum biochemistry and complete blood panel analysis, which were performed in Drug Safety Evaluation Research Center (Shanghai Institute of Materia Medica, Shanghai, China). Three mice were included for each group.

\subsection{Statistical Analysis}

Statistical analysis was performed using GraphPad Prism 8.0 software (La Jolla, CA). Differences between groups were assayed using Student's ttest or ANOVA with Tukey's multiple comparison tests. The $\mathrm{p}$ value below 0.05 was considered significant.

\section{Results and Discussion}

\subsection{Preparation and Characterization of Targeted Micellar Phthalocyanine (T-MP)}

Pluronic F127, an FDA-approved non-ionic copolymer widely used for drug delivery [33], was adopted for the preparation of micellar phthalocyanine as previously described [19]. For trastuzumab modification, the terminal hydroxyl group of F127 was carboxylated via reaction with succinic anhydride [25]. A $\mathrm{C}=\mathrm{O}$ stretching signal at $1737.3 \mathrm{~cm}^{-1}$ in the FTIR spectrum indicated successful carboxylation of Pluronic F127 (Fig. S1).

Empty F127 micelles were formed through dispersing F127 (15\% carboxylation) in water $(10 \% \mathrm{w} / \mathrm{v})$. Then, $2 \mathrm{mg}$ VBPc (structure shown in Fig. S2) in $1 \mathrm{~mL}$ dichloromethane was added. The mixture was stirred until the dichloromethane was evaporated to generate VBPc-loaded F127 micelles. The micelles were then purified via CMC switching and centrifugal filtration at $4{ }^{\circ} \mathrm{C}$ to remove the empty micelles and loosely bound F127 to provide the resulting concentrated micellar phthalocyanine (MP) [19]. Trastuzumab was then linked to MP surface via EDC/NHS chemistry to obtain targeted micellar phthalocyanine (T-MP). Trastuzumab modification conferred two amide peaks at $1644 \mathrm{~cm}^{-1}$ and $1563 \mathrm{~cm}^{-1}$ in the FTIR spectra (Fig. S1) and also increased surface nitrogen (17.9\%) compared to that (3.7\%) of the unmodified MP identified by X-ray photoelectron spectroscopy (XPS) (Fig. S3).

Unmodified MP was $20 \mathrm{~nm}$ spheres, and antibody modification (T-MP) increased the size to $40 \mathrm{~nm}$ as observed 
in transmission electron microscopy (TEM) (Figs. 1a and S4). This size change was also confirmed in the dynamic light scattering (DLS) (Fig. 1b), showing a hydrodynamic size of $48 \mathrm{~nm}$ for T-MP versus $18.6 \mathrm{~nm}$ for unmodified MP. Trastuzumab modification decreased the nanoparticle surface charge from -25.3 to $-13.2 \mathrm{mV}$ (Fig. 1c), which may be ascribed to the depletion of the carboxyl groups of the F127 micelles.

Non-reducing sodium dodecyl sulfate-polyacrylamide gel electrophoresis (SDS-PAGE) with coomassie blue staining assay was also performed to examine the antibody modification. Compared to the free antibody, T-MP moved slightly more slowly due to the increased size, although the molecular weight of F127 (12.7 kDa) is significantly less than the antibody ( 150 kDa) (Fig. 1D). VBPc fluorescence in the micelles was largely selfquenched due to their aggregation in micelle core (Fig. S5). For micelle fluorescent labeling, Cy5.5-NHS ester that could be reacted with the antibody amine groups was incubated with T-MP to generate Cy5.5-labeled T-MP. The corresponding single Cy5.5 signal band indicated the successful fluorescent labeling, high T-MP purity, and high conjugation efficiency ( 100\%) of antibodies (Fig. 1D). We further determined the number of antibodies on each nanoparticle. Through BCA assay, the antibody loading in T-MP was estimated to be $1.5 \%$. The weight of each T-MP particle $(\mathrm{m})$ was calculated using the equation: $m=\rho^{*}\left(\pi \mathrm{D}^{3} / 6\right)$ [34]. In this equation, $\rho$ was the nanoparticle weight per volume unit (density), estimated to be $1 \mathrm{~g} \mathrm{~cm}^{-3}$. D was the number-based mean nanoparticle diameter $(48 \mathrm{~nm})$ determined by DLS. Thus, the number of antibodies on each nanoparticle was estimated to be 28 .

Free VBPc had a maximum absorption peak at $808 \mathrm{~nm}$, while the micellar VBPc had absorption peaks of comparable intensity at 736 and $790 \mathrm{~nm}$ (Fig. 1e), consistent with previous reports [19]. After disrupting the lyophilized micelles with DMSO, VBPc loading in T-MP was determined to be $69.1 \%$ using the optical absorbance at $800 \mathrm{~nm}$. This significantly high drug loading, compared to that (typically below 5\%) [35] produced using the conventional method without stripping process, reflected the advantage of micelle production using the surfactant-stripping strategy [36, 37]. High drug loading would generally decrease the excipient-associated side effect and may also enable higher dose of drug to be used, which can be useful when drugs need to be administered in a smaller volume [36]. Upon $808 \mathrm{~nm}$ light irradiation $\left(2.0 \mathrm{~W} \mathrm{~cm}^{-2}, 5 \mathrm{~min}\right)$, micellar VBPc in water exhibited concentration-dependent $\left(10-100 \mu \mathrm{g} \mathrm{mL}^{-1}\right)$ temperature increase to $79.9{ }^{\circ} \mathrm{C}$ (Figs. 1f and S6). Laser power (1-2.5 W) -dependent temperature elevation of T-MP $\left(20 \mu \mathrm{g} \mathrm{mL}^{-1} \mathrm{VBPc}\right)$ was also characterized (Figs. 1g and S7). T-MP demonstrated good photothermal stability; after 20 min laser irradiation, the optical absorption spectrum and intensity remained nearly unchanged (Fig. 1h). Also, three consecutive cycles of NIR-induced heating achieved the similar and comparable temperature increase patterns (Fig. 1i). The photothermal conversion efficiency of T-MP was calculated to be $16.8 \%$, which was comparable to that of Au nanorod (17\%) [17], but was lower than the emerging semiconducting polymer nanoparticles $(27.5 \sim 71 \%)$ [17, 27, 38, 39]. Micellar VBPc also possessed strong photoacoustic (PA) imaging properties (Fig. 1j), which can be used to identify the distribution in the metastatic LN, as well as for photothermal contrast. No obvious differences were observed with respect to the optical properties of nanoparticles before and after antibody conjugation (Fig. S8), indicating the minimal influence of antibody conjugation on the micellular core where VBPc located. T-MP was colloidally stable in PBS at $4{ }^{\circ} \mathrm{C}$ for 7 days, PBS with $10 \%$ FBS at $37{ }^{\circ} \mathrm{C}$ for $48 \mathrm{~h}$, and McCoy's $5 \mathrm{~A}$ medium at $37^{\circ} \mathrm{C}$ for $48 \mathrm{~h}$ with the size remained almost unchanged (Fig. S9).

\subsection{Enhanced Uptake and Photothermal Cytotoxicity of T-MP in HT-29 Cells}

HER2 is a clinically relevant therapeutic target for multiple cancers, including breast, gastric, and lung cancer [40]. HER2 is also found to be involved in the progression of some colorectal cancers, and anti-HER2 therapy against colorectal cancer is undergoing in clinical trials $[41,42]$. Indeed through immunohistochemical assay of the tumor tissues from 13 colorectal cancer patients, we also identified that HER2 expression in tumor cells was higher than that in the tumor stroma and adjacent normal tissues (Figs. 2a and S10). These observations suggest that the HER2 receptor may be a clinically relevant target, at 
(a)
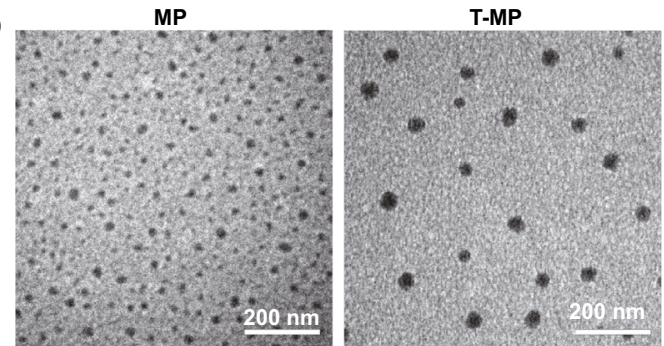

(b)

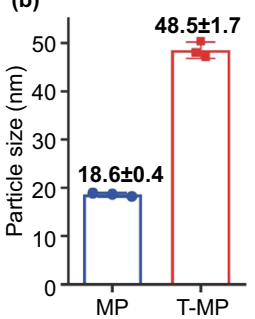

(f) (c)

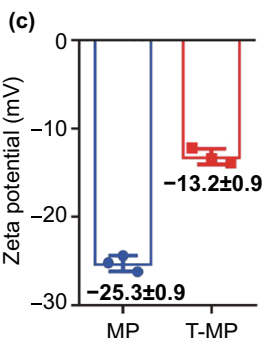

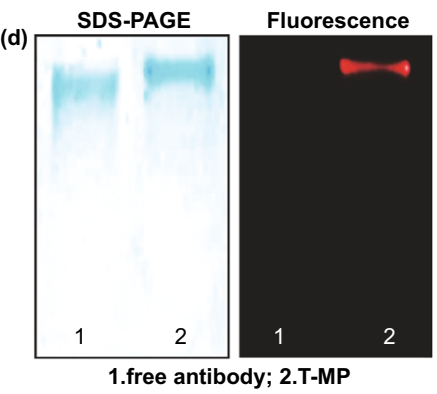

1.free antibody; 2.T-MP
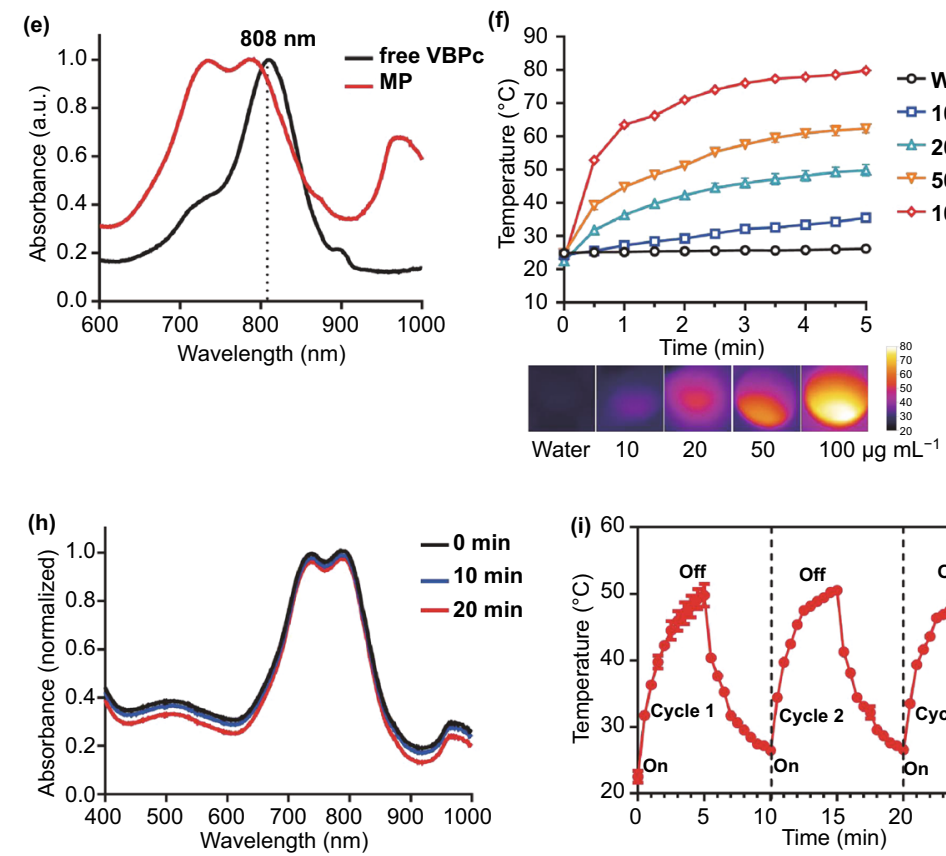

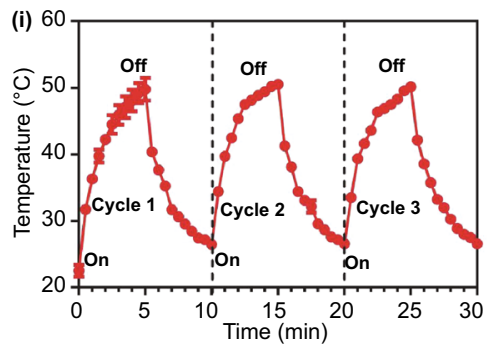

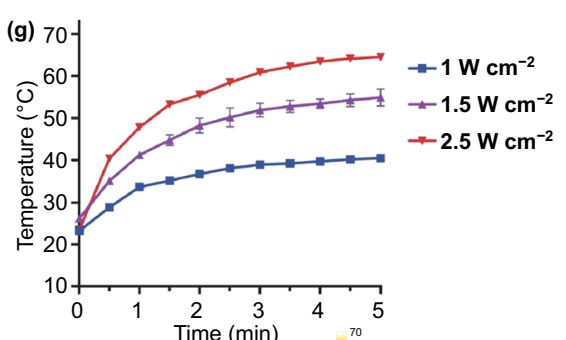
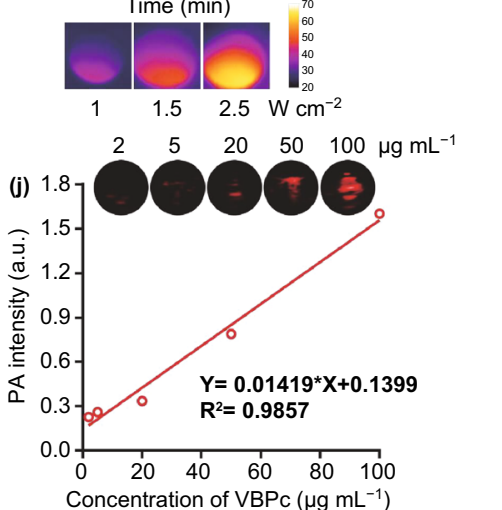

Fig. 1 Characterization of micellular VBPc. a TEM images of MP (unmodified) and T-MP. Particle size (b) and zeta potential (c) determined by dynamic light scattering (DLS). d SDS-PAGE with coomassie blue staining (left) and near-infrared fluorescence imaging (right) of free antibody and T-MP. e Vis-NIR absorbance of free VBPc in dichloromethane and unmodified MP in water. $\mathbf{f}$ VBPc concentration-dependent temperature increases of T-MP at power density of $2 \mathrm{~W} \mathrm{~cm}^{-2}$. Representative photothermal images at 5 min were captured by thermal imaging camera (Testo 890) $\mathbf{g}$ Light power-dependent temperature increase curves of T-MP $\left(20 \mu \mathrm{g} \mathrm{mL} \mathrm{m}^{-1} \mathrm{VBPc}\right)$. Representative photothermal images at $5 \mathrm{~min}$ were shown. $\mathbf{h}$ Consecutive cycles of laser-induced heating of T-MP (VBPc $20 \mu \mathrm{g} \mathrm{mL}^{-1}$ ) under $808 \mathrm{~nm}$ irradiation at $2 \mathrm{~W} \mathrm{~cm}$. $\mathbf{i}$ Influence of irradiation time on the Vis-NIR absorption curve of T-MP. $\mathbf{j}$ PA imaging property of T-MP. Data are presented as mean \pm s.d. $(n=3)$

least for a portion of patients, for colorectal cancer-targeted drug delivery. HT-29, a human colorectal cancer cell line with strong HER2 expression (Fig. 2b) [22, 23], was used in this study. Micelles labeled with iFluor 594 were used for the cell uptake assay. Compared with nontargeted isotype lgG-conjugated micelles (nonT-MP), trastuzumab modification (T-MP) enhanced cellular uptake by $2 \sim 4$ times in a time-dependent manner (Fig. 2c, d). This effect would lead to enhanced photothermal cytotoxicity to HT-29-luc cells as illustrated in Fig. 2e. In another test, HER2-negative 4T1 cells were also used for cellular uptake assay (Fig. S11). No differences were observed between the uptake of T-MP and nonT-MP in 4T1 cells. This evidence together with the observation in HT-29 cells demonstrated the specific targeting ability of T-MP in HER2-positive cells.

For photothermal therapy in vitro, HT-29-luc cells were treated with micellular VBPc then subjected to 
(a)

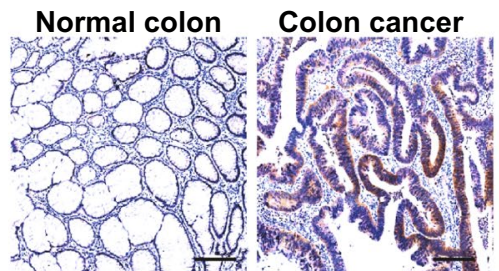

(c)

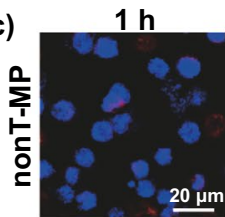

$1 \mathrm{~h}$

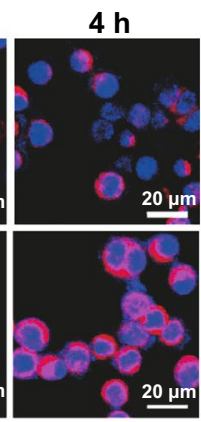

(f)

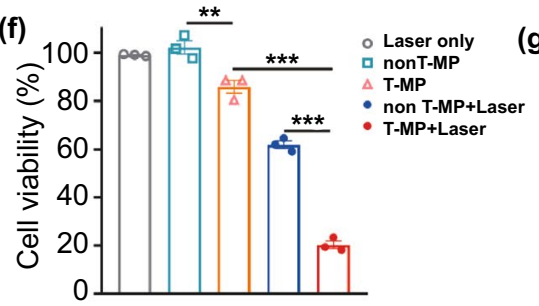

(b)

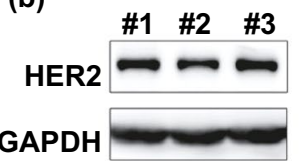

(d)

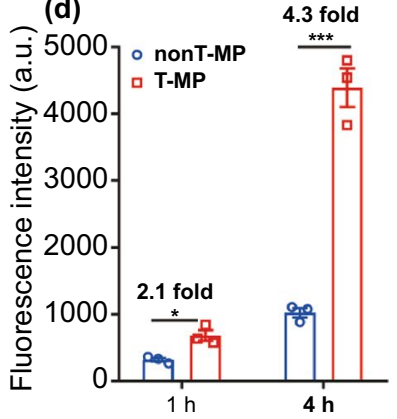

(g)

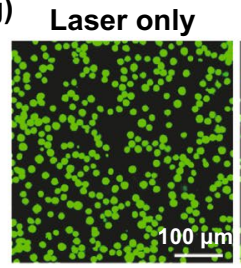

(e) $808 \mathrm{~nm}$

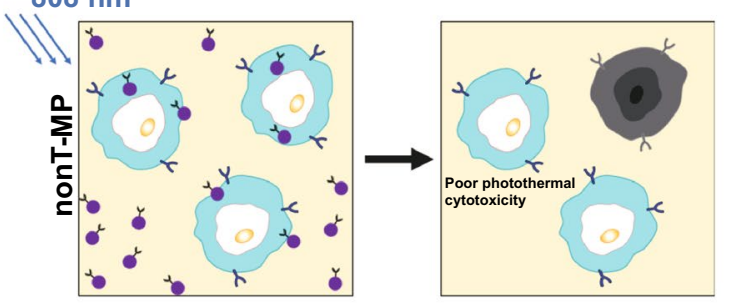

$808 \mathrm{~nm}$

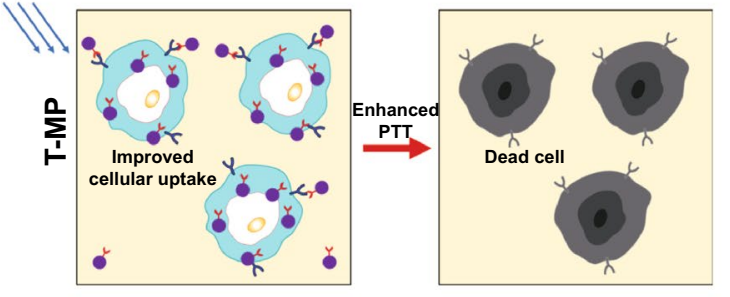

Fig. 2 Targeted cellular uptake and improved photothermal-induced cytotoxicity. a Representative immunohistochemical staining showing high HER2 expression in colon cancer from human patients, compared to human normal colon tissues. b Western blot analysis for HER2 expression in HT-29 cells. \#1, \#2, and \#3 are three replicated samples. GAPDH (glyceraldehyde-3-phosphate dehydrogenase) was used as control. c Confocal fluorescence images of cellular uptake in HT-29-luc cells after 1 and 4 h-incubation with iFluor 594-labeled micelles $\left(E_{\mathrm{x}} 563 \mathrm{~nm}, E_{\mathrm{m}}\right.$ $604 \mathrm{~nm}$ ). d iFluor 594 fluorescence intensity in HT-29 cells analyzed by ArrayScan XTI High Content Analysis Reader. e Illustration showing how improved cellular uptake conferred by targeted antibody modification enhances photothermal-induced cytotoxicity. f Cell viability after treatment with micelles and laser irradiation. Micelles or laser alone were included as control. $\mathrm{g}$ Fluorescence microscope images of calcein$\mathrm{AM} / \mathrm{PI}$ co-stained HT-29-luc cells after various treatments. Live and dead cells were shown in green (Calcein-AM; $E_{\mathrm{x}} 490 \mathrm{~nm}, E_{\mathrm{m}} 515 \mathrm{~nm}$ ) and red (PI; $\left.E_{\mathrm{x}} 530 \mathrm{~nm}, E_{\mathrm{m}} 580 \mathrm{~nm}\right)$, respectively. Data are presented as mean \pm s.d. $(n=3) . * p<0.05, * * p<0.01, * * * p<0.001$

$808 \mathrm{~nm}$ laser irradiation. As expected, the photothermal killing effect caused by T-MP (only $20.3 \%$ viable cells left) was stronger than that of nonT-MP $(61.9 \%$ cell viability) (Fig. 2f). No cytotoxicity occurred to the cells treated with nonT-MP alone or laser alone. However, trastuzumab attached to the micelle surface exhibited moderate killing effect on HT-29 cells; this can be ascribed for the blockage of the HER2 signaling pathway [40].

The improved photothermal therapy conferred by T-MP was further confirmed by calcein-AM and propidium iodide (PI) dual-staining tests (Fig. 2g). The least live (green) and most dead (red) cells after T-MP targeted photothermal treatment indicated the strongest cytotoxic killing.

\subsection{Orthotopic Colorectal Cancer Model and Targeted Accumulation of T-MP in Metastatic Sentinel LNs}

With injection of tumor cells into submucosa of cecum, the orthotopic HT-29-luc colorectal cancer model was established (Fig. 3a, b) [24]. HT-29-luc tumor growth was accompanied by LN metastasis that mostly occurred in the mesentery as illustrated in Fig. 3c. Pathological and 
immunohistochemical analysis showed that the detectable metastases were usually solitary and located in the LN cortex region (Fig. S12).

Through sensitive bioluminescence imaging showing that as least as 100 tumor cells can also be detected (Fig. S13), we identified the LN metastasis pattern with time. Within 15-36 days after tumor cell inoculation, LN metastasis only occurred to the mesenteric LN, but not the pancreaticoduodenal LNs, and the lumbar LNs (Fig. S14). It is noted that during day 15 to 22 , metastasis only happened to the sentinel LNs, but not the next ones following the flow of LN direction. Meanwhile, no organ metastasis appeared within 22 days after tumor cell inoculation (Fig. S15). Therefore, mice with tumor cell inoculation for 15-22 days were used in this study.

It has been well demonstrated that small-sized nanoparticles $(<50 \mathrm{~nm})$, but not large ones, can effectively enter into the LN metastases to enable therapy [7]. Based on this finding, we here explored whether such nanoparticle accumulation in LN metastasis could be further improved by endowing the nanocarriers with the targeting property for tumor cells. Mice bearing orthotopic tumors and LN metastases were then intravenously administered with Cy5.5labeled T-MP. As demonstrated in Fig. 3d, T-MP successfully targeted to the metastatic mesenteric sentinel LN as demonstrated by sequential bioluminescence and fluorescence imaging in situ or ex vivo. We further examined the time-dependent distribution of T-MP in the metastatic LNs (Fig. 3e, f). The accumulation of T-MP increased with the time and reached its peak at $8 \mathrm{~h}$ after injection. Importantly, the targeting efficacy of T-MP in the metastatic sentinel LNs was markedly higher than that of nonT-MP, exhibiting 2.2and 4.4-folds increased distribution at 4 and $8 \mathrm{~h}$, respectively. This advantage may be ascribed to the small micelle size and the improved tumor cell targeting and uptake conferred by the conjugated anti-HER2 antibody (Fig. 2c, d).

The biodistribution of the micelles and the cargo (VBPc) at $8 \mathrm{~h}$ after injection was examined. The accumulation of T-MP in tumor tissue was higher than that of nonT-MP (Fig. S16), and same observation was obtained for the VBPc contents in the tumors (Fig. S17). There was no significant difference in their distribution in other organs. To examine whether the enhanced distribution in the primary tumor contributed to the improved accumulations in metastatic sentinel
$\mathrm{LN}$, in another test, the orthotopic tumor was in advance excised before the micelle injection. It showed that the distribution of T-MP in metastatic sentinel LNs was comparable to that without primary tumor resection, and higher than that of nonT-MP (Figs. S18 and 3e, f). Moreover, the micelle distribution in healthy LNs was obviously much lower. These observations were consistent with the literature, demonstrating that metastatic LN targeting by small-sized nanoparticles was less relevant to primary tumor [7]. This improved micelle distribution led to elevated VBPc contents in metastatic sentinel LNs (Fig. 3g), which would confer stronger photothermal effects when light irradiation was performed.

We further performed immunofluorescence staining of the metastatic LNs to investigate the micro-distribution of micelles in the LNs and the targeting of Cy5.5-labeled micelles (red) to the metastatic foci (anti-HER2 staining, green) (Fig. 4a, b). The micelles were found to be mainly distributed in the metastasis region, and the accumulation of T-MP in metastasis region was 3.0-folds higher than that of nonT-MP micelles (Fig. 4c). There was no significant difference between the low-distribution of the two micelles in the non-metastasis region of the LNs. Noted that the accumulation of nonT-MP in metastasis region was 1.6-fold higher than that in the non-metastasis region, demonstrating the LN metastasis targeting capacity of small-sized particles [7].

PA imaging also showed that compared with nonT-MP, the PA signal of T-MP in metastatic LNs was much higher with around 6 times enhancement (Fig. 4d). Noted that the PA signal was unevenly distributed in the LNs, but specifically distributed more on one side of the LNs. This phenomenon was consistent with the observation that the metastatic foci were located at peripheral cortex region of the LNs (Fig. S12).

\subsection{Photothermal Therapy of Metastatic LNs and Antitumor Efficacy}

We next investigated the benefit of photothermal therapy of metastatic LNs conferred by T-MP over nonT-MP. The HT29-luc tumor-bearing mice were i.v. injected with T-MP or nonT-MP $\left(5 \mathrm{mg} \mathrm{kg}^{-1} \mathrm{VBPc}\right)$. After $8 \mathrm{~h}$, the orthotopic tumor was surgically resected, and the sentinel $\mathrm{LN}$ was irradiated with $808 \mathrm{~nm}$ laser $\left(2 \mathrm{~W} \mathrm{~cm} \mathrm{~cm}^{-2}, 10 \mathrm{~min}\right)$ for thermal ablation of the metastatic tumor cells. The experimental procedure 
(a)

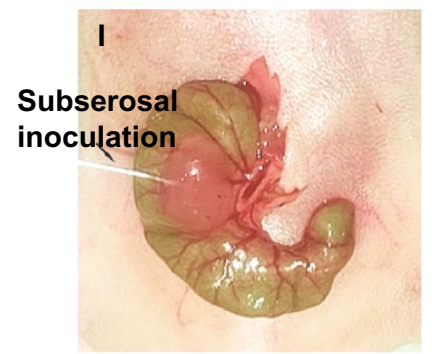

(d)

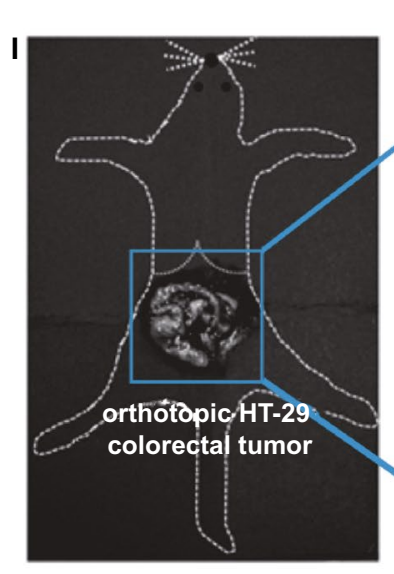

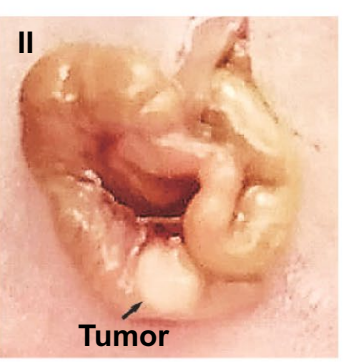

Before
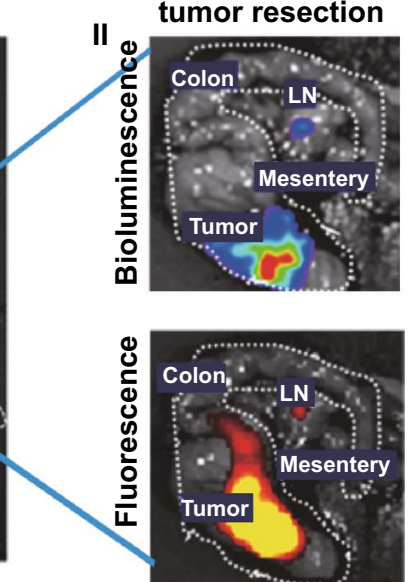

(b)

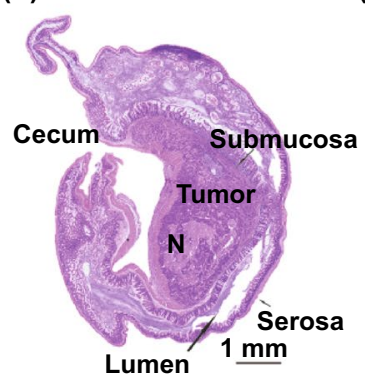

After tumor resection

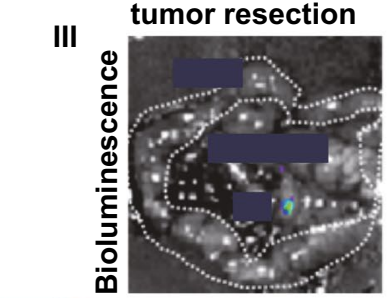

$\rightarrow$

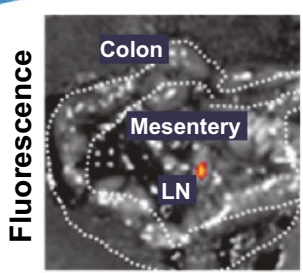

(f)

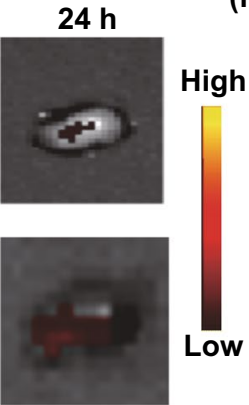

(c)

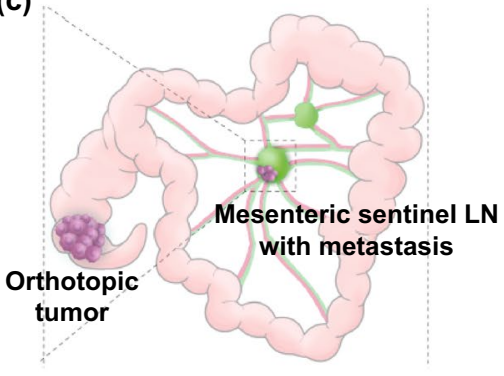

Resected metastatic LN

IV
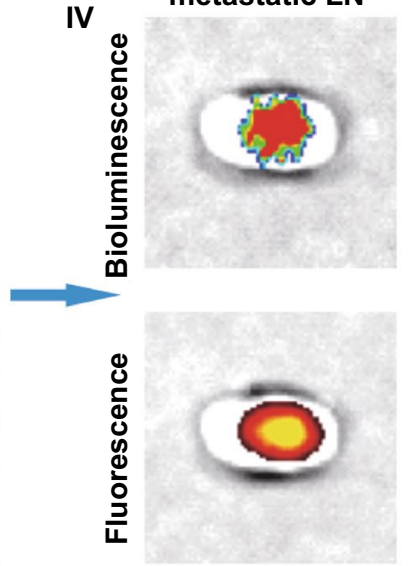

(e)
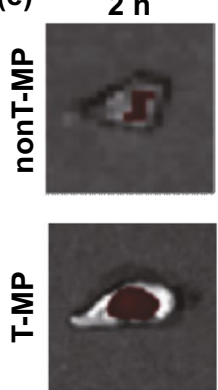

$4 \mathrm{~h}$
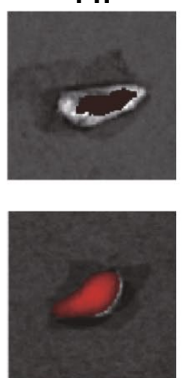

$8 \mathrm{~h}$
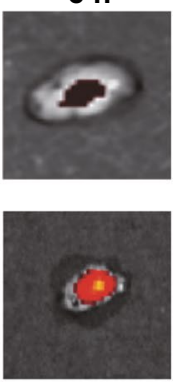
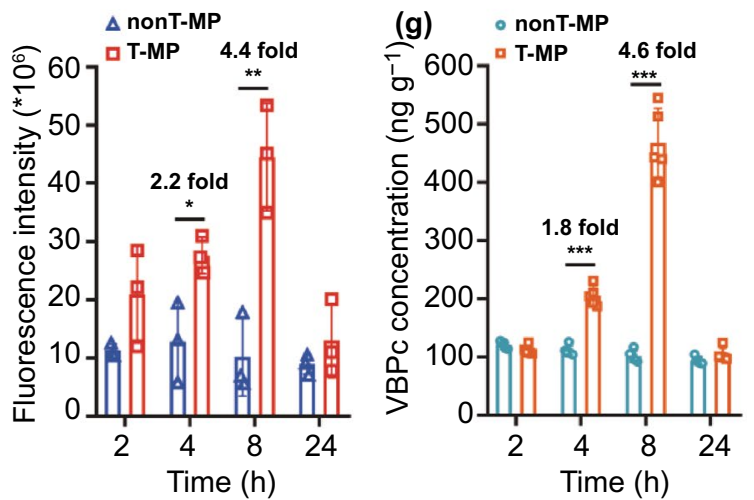

Fig. 3 Orthotopic HT-29-luc colorectal cancer model and metastatic LN targeting of T-MP. a Establishment of orthotopic HT-29-luc tumor by sub-serosal injection of tumor cells in the cecum (I). The solid tumor was visible to the naked eye after 2 weeks (II). b Hematoxylin and eosin (H\&E) staining showed the right anatomical location of the orthotopic tumor. c Illustration of the metastatic sentinel LN in the mesentery. $\mathbf{d}$ The metastatic mesenteric sentinel LN was targeted and imaged using Cy5.5-labeled T-MP after intravenous administration. (I), Illustration of the orthotopic HT-29-luc tumor in mouse abdominal cavity. (II), Bioluminescent and fluorescent imaging of the orthotopic tumor and metastatic sentinel LN. (III), Bioluminescent and fluorescent imaging of the metastatic sentinel LN after tumor resection. (IV), Ex vivo imaging of the excised metastatic sentinel LN. e Time-dependent, ex vivo imaging of the Cy5.5-labeled micelles in the metastatic sentinel LNs. f Quantified fluorescence intensity in panel E. g VBPc contents in metastatic sentinel LN. Data are presented as mean \pm s.d. $n=3$ in panel F and 5 in panel G. $* p<0.05, * * p<0.01, * * * p<0.01$ 
(a)
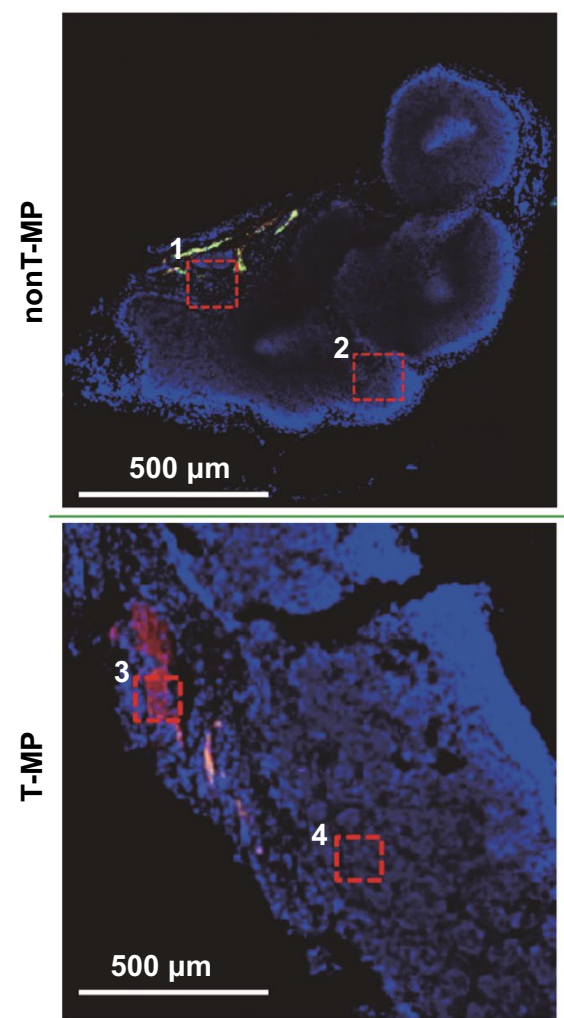

(c)

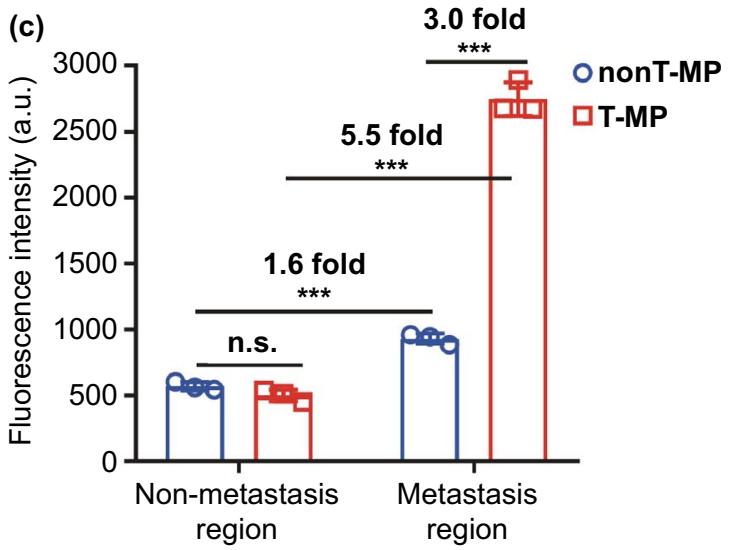

(b) Hoechst 33342
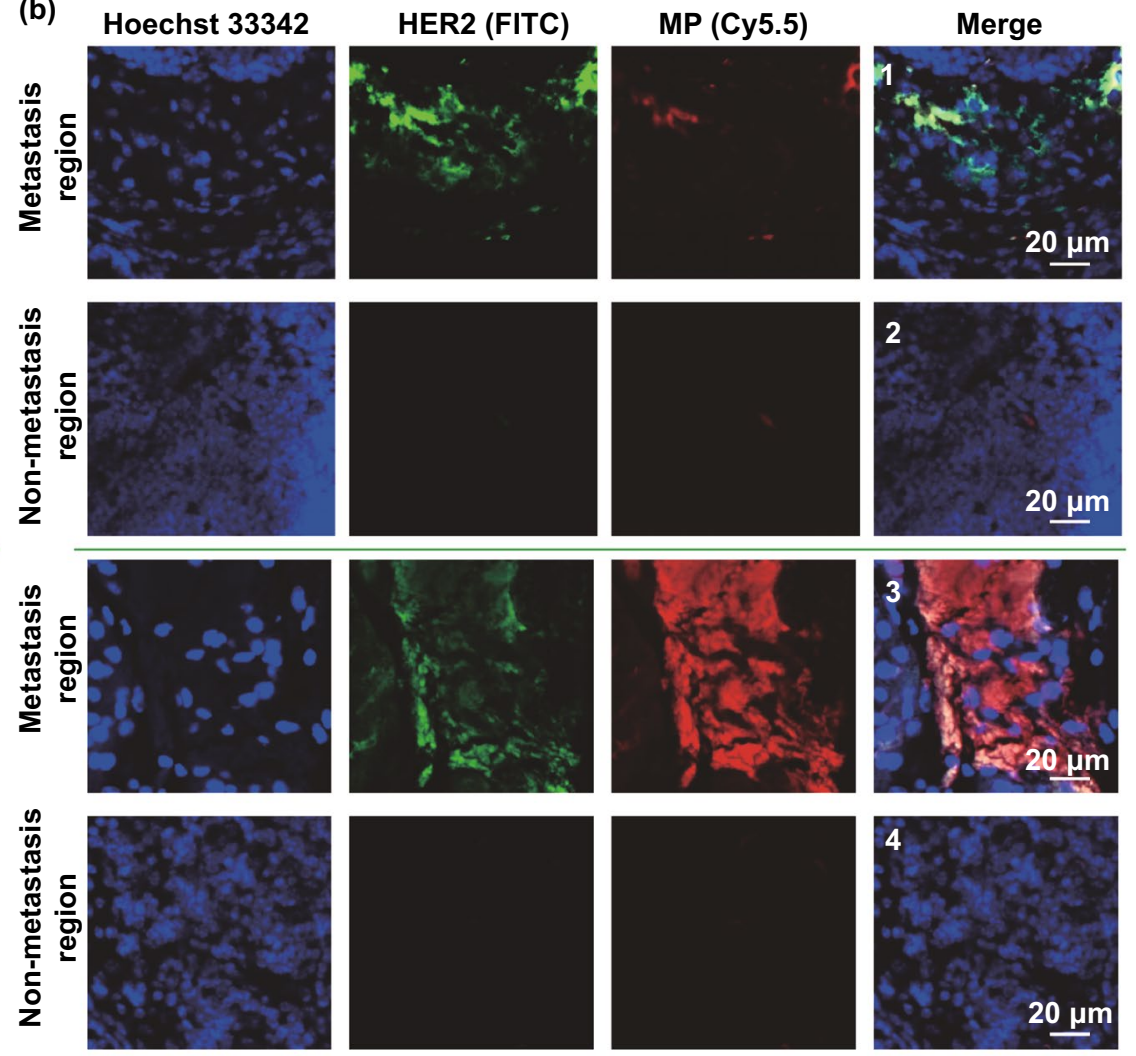

(d)
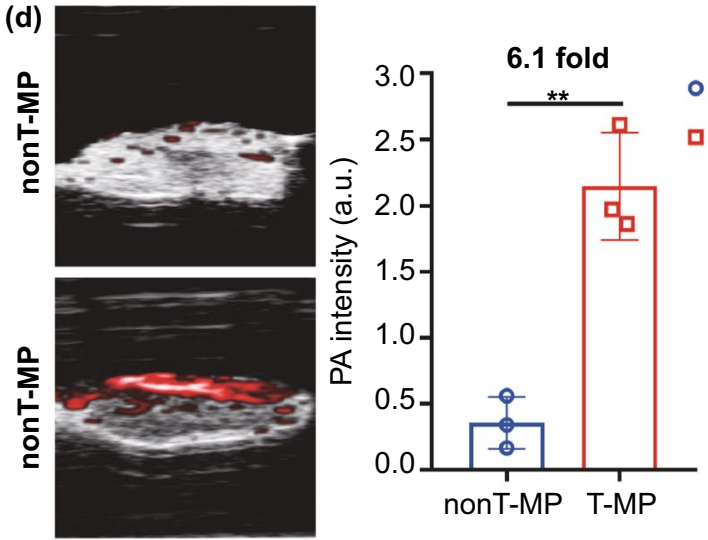

Fig. 4 Micro-distribution of the micelles in the metastatic sentinel LNs and LN PA imaging. a Immunofluorescent staining of the metastatic sentinel LNs $8 \mathrm{~h}$ after i.v. injection of the T-MP or nonT-MP. The micelles were labeled with Cy5.5 (red) and the metastatic tumor cells were stained with rabbit anti-HER2 antibody and FITC goat anti-rabbit antibody (green). The region with metastasis (1 and 3) and metastasis-free region ( 2 and 4 ) was enlarged in panel (b). c Quantified fluorescence intensity in panel B. n.s., not significant. d Ex vivo PA imaging of the excised metastatic sentinel LNs. Quantified PA signals were compared. Data are presented as mean \pm s.d. $(n=3)$. $* * p<0.01, * * * p<0.001$ 
is illustrated in Fig. 5a. The temperature of the sentinel LN treated with T-MP increased from 27.3 to $54.3{ }^{\circ} \mathrm{C}$, pronounceably higher than that $\left(46.5^{\circ} \mathrm{C}\right)$ treated with nonTMP (Fig. 5b). This observation could be ascribed to the improved distribution of T-MP compared to nonT-MP in the metastatic LN (Fig. 3e-g). Moreover, this higher temperature might cause enhanced killing to the tumor cells in the LN. After $48 \mathrm{~h}$, the irradiated sentinel LNs were excised and processed for electron microscopic observation. More dead tumor cells characterized by cell shrinkage, nuclear cracking and pyknosis were observed in the metastatic LNs treated with T-MP, but not nonT-MP, assisted photothermal therapy (Fig. 5c).

The untreated mice or the mice treated with tumor resection only or together with the micelles but no laser irradiation were included as controls. The growth or recurrence of the tumors and the mice survival as well as body weight were monitored and recorded. It showed tumor recurrence was dramatically delayed and suppressed when the metastatic LNs were treated with T-MP-assisted laser irradiation (Fig. 5d, e). Accordingly, mice life span was dramatically extended with median survival of $96 \mathrm{~d}$, earning a biggest increase in life span (ILS) of $113.3 \%$, compared with other controls (Fig. 5f, Table S1). It is noted that this therapeutic outcome was comparable to or even better than the traditional treatment of tumor resection plus lymphadenectomy, which earned a 91-day median survival (Fig. S19), demonstrating the advantage of this local and minimally invasive treatment. In contrast, lymphadenectomy usually causes several risks and complications such as bleeding, infection, pain, phlebitis, nerve injury, and lymphedema, which compromise the quality of life [43].

To determine the cause of mouse death, we performed autopsy of the mice from T-MP + Laser group through bioluminescence imaging. The luciferase in the tumor cells was still active shortly after the mouse death. For bioluminescence imaging, the resected organs were previously soaked with the PBS containing luciferin, $\mathrm{Mg}^{2+}$, and ATP for $5 \mathrm{~min}$ as we described [44]. Tumor recurrence in situ (intestine) or the metastases in liver and lung were observed in the mice of both T-MP+Laser group (Fig. S20) and nonT-MP+Laser group (Fig. S21), which would be responsible for the animal death. The metastases in the organs were also confirmed using hematoxylin and eosin (H\&E) staining (Figs. S22 and S23).

Following treatment, the mouse body weights were well maintained (Fig. 5g), indicating the tolerance of the treatment. Pathological examination of the organs, including heart, liver, spleen, lung, and kidney in all groups showed no significant histological toxicity (Fig. S24). Blood analysis was also performed for the treatment-associated toxicity evaluation. Key parameters reflecting liver and kidney function and hematological toxicity were examined after 24, 96, and $168 \mathrm{~h}$ (Figs. S25-S27). It showed no obvious difference in the parameters of T-MP plus laser group, when compared to other controls, indicating the low toxicity of the therapy.

\section{Conclusions}

In summary, this study proposed a new method to improve the targeting and treatment of LN metastases. Through introducing targeting capacity, small-sized nanoparticles $(<50 \mathrm{~nm})$ accumulated more in LN metastasis than the nontargeted counterparts, as proved using a metastatic orthotopic colorectal tumor model. This improved targeting could be used for enhanced therapy such as local and minimally invasive photothermal therapy to overcome LN metastasis and suppress disease recurrence, eventually leading to a prolonged animal survival. Moreover, the improved therapeutic outcome of the metastatic LNs was comparable or even better than the traditional lymphadenectomy that usually incurred surgical trauma and postoperative complications. Our findings exhibited the potential of targeted small-sized nanoparticles to enable superior paradigms for dealing with LN metastases. 

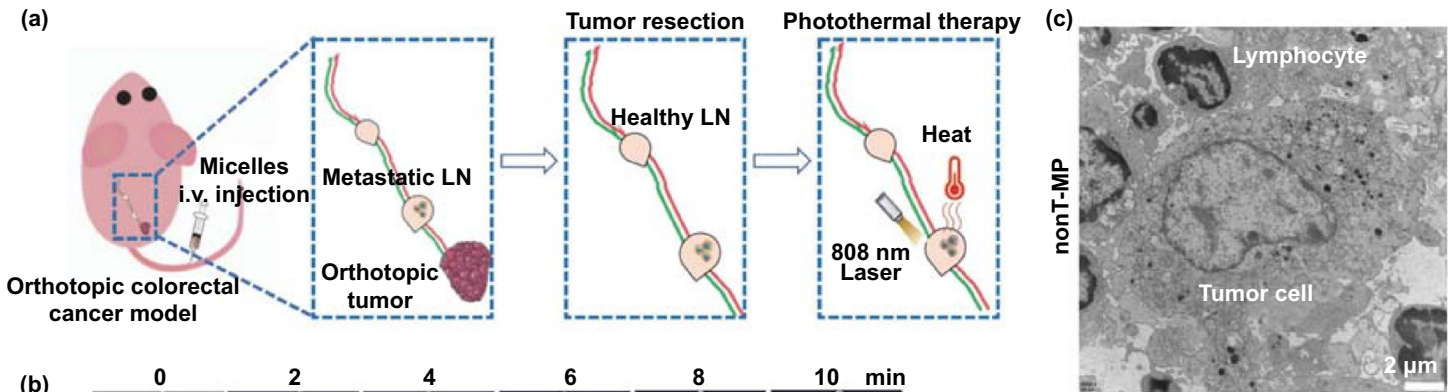

(b)
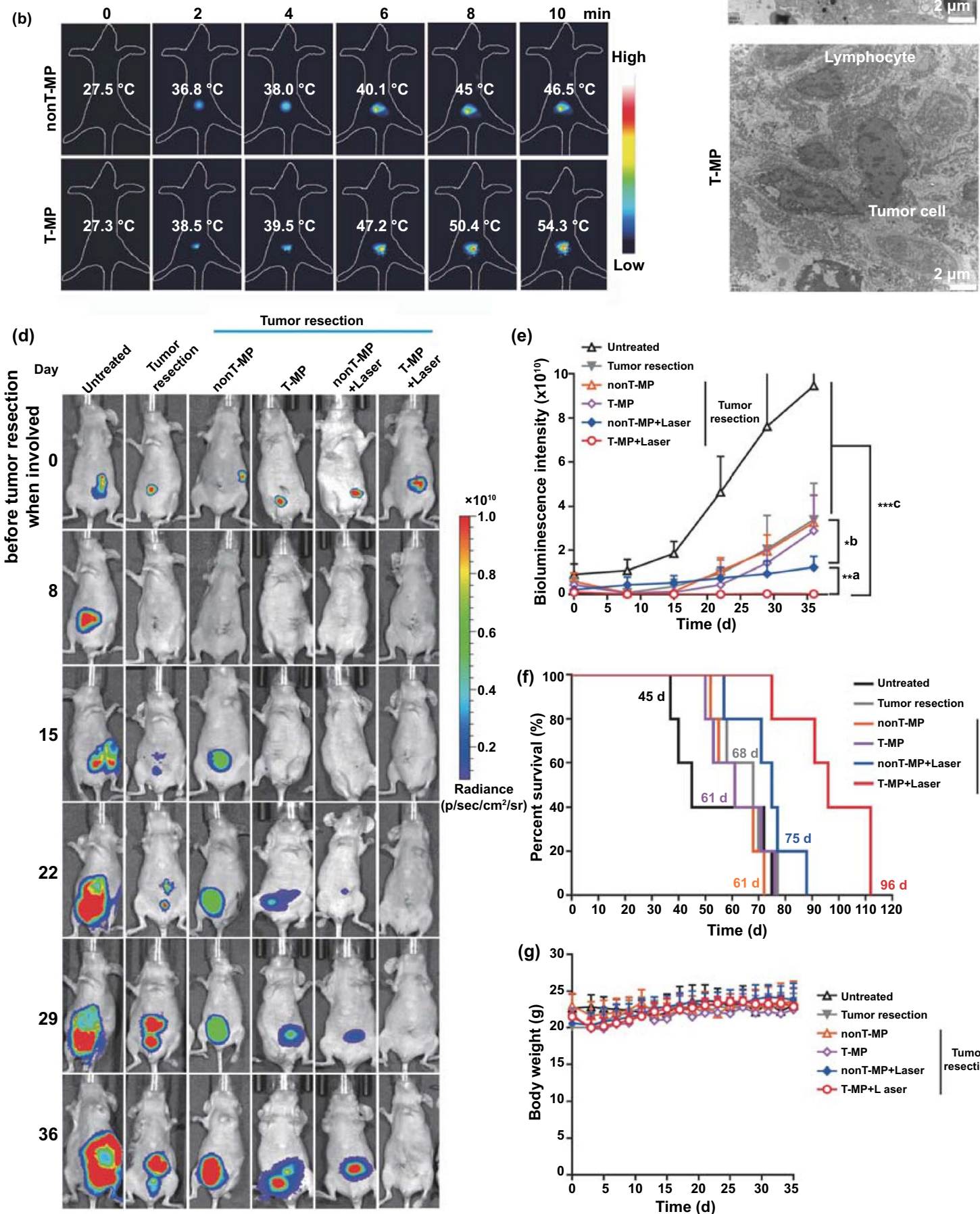

(e)
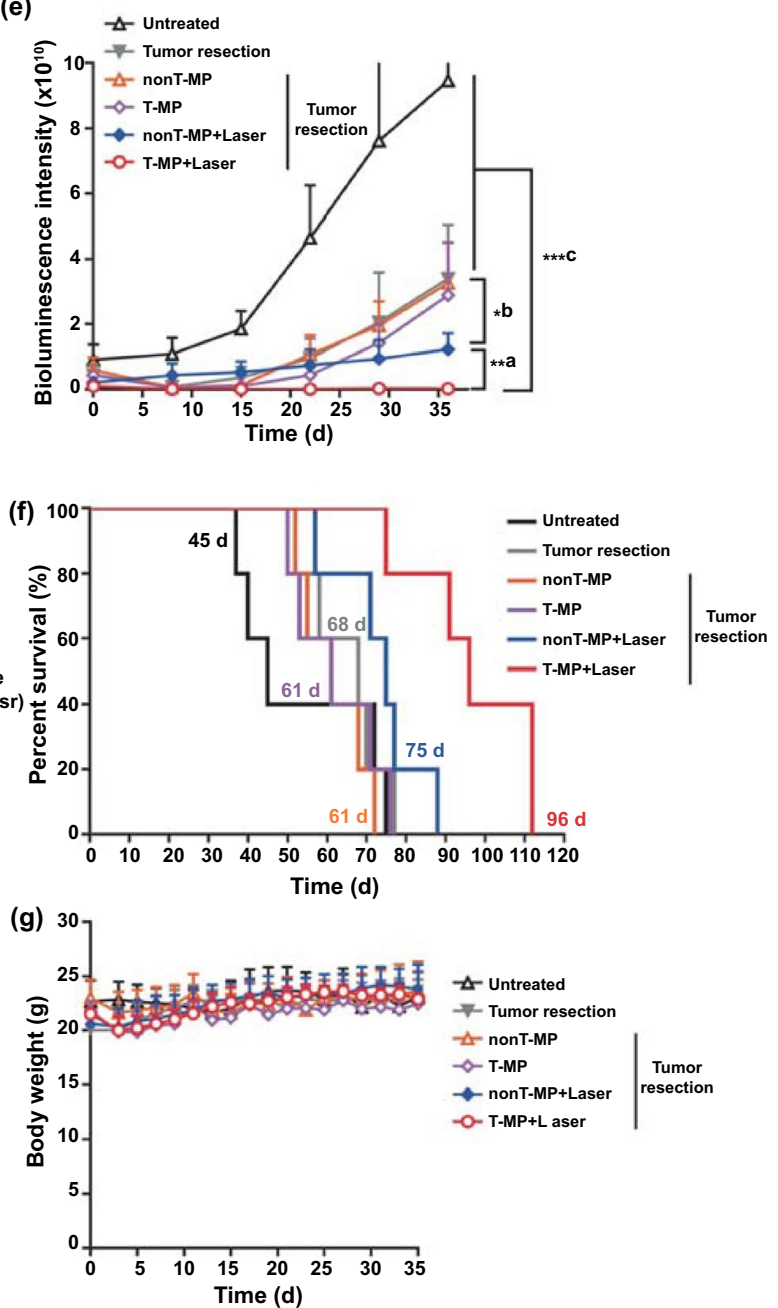
\Fig. 5 Photothermal therapy of metastatic sentinel LNs and antitumor evaluation. a Illustration of the treatment of the surgical resection of orthotopic tumor and follow-up laser irradiation of the metastatic LNs on day 0 . b Temperature increase of the metastatic LNs was recorded using Testo 890 thermal imager. c Representative TEM images of LN sections after photothermal therapy. d Representative bioluminescence imaging of the mice before tumor resection (day 0 ) and after tumor resection and photothermal therapy over the time. e Quantified bioluminescence intensity in panel D. (1) T-MP+Laser versus nonT-MP+Laser; (2) nonT-MP+Laser versus T-MP, nonTMP, and Tumor resection. (3) T-MP+Laser versus all other controls except nonT-MP + Laser. f Survival of the mice with indicated treatments. The median survivals of the mice were noted. g Mice body weight. Data are presented as mean \pm s.d. $(n=5) . * p<0.05$, $* * p<0.01$, and $* * * p<0.001$

Acknowledgements Hai-Yi Feng and Yihang Yuan contributed equally to this work. We thank Prof. Gang Zheng (University of Toronto) for valuable discussion. We also thank the Core Facility of Basic Medical Sciences (SJTU-SM) for frozen section making and scanning. This work was supported by National Natural Science Foundation of China (81572998, 81773274, 82073379), Shanghai Municipal Science and Technology Commission (20ZR1451700, 16520710700), and Shanghai Collaborative Innovation Center for Translational Medicine (TM201731).

Open Access This article is licensed under a Creative Commons Attribution 4.0 International License, which permits use, sharing, adaptation, distribution and reproduction in any medium or format, as long as you give appropriate credit to the original author(s) and the source, provide a link to the Creative Commons licence, and indicate if changes were made. The images or other third party material in this article are included in the article's Creative Commons licence, unless indicated otherwise in a credit line to the material. If material is not included in the article's Creative Commons licence and your intended use is not permitted by statutory regulation or exceeds the permitted use, you will need to obtain permission directly from the copyright holder. To view a copy of this licence, visit http://creativecommons.org/licenses/by/4.0/.

Supplementary Information The online version contains supplementary material available at https://doi.org/10.1007/ s40820-021-00666-8.

\section{References}

1. S.A. Stacker, S.P. Williams, T. Karnezis, R. Shayan, S.B. Fox et al., Lymphangiogenesis and lymphatic vessel remodelling in cancer. Nat. Rev. Cancer 14, 159-172 (2014). https://doi. org/10.1038/nrc3677

2. E.R. Pereira, D. Kedrin, G. Seano, O. Gautier, E.F.J. Meijer et al., Lymph node metastases can invade local blood vessels, exit the node, and colonize distant organs in mice. Science 359, 1403-1407 (2018). https://doi.org/10.1126/science.aal36 22
3. P. Harter, J. Sehouli, D. Lorusso, A. Reuss, I. Vergote et al., A randomized trial of lymphadenectomy in patients with advanced ovarian neoplasms. N. Engl. J. Med. 380, 822-832 (2019). https://doi.org/10.1056/NEJMoa1808424

4. E. Van Cutsem, X. Sagaert, B. Topal, K. Haustermans, H. Prenen, Gastric cancer. Lancet 388, 2654-2664 (2016). https:// doi.org/10.1016/S0140-6736(16)30354-3

5. I.G. Kwon, T. Son, H.I. Kim, W.J. Hyung, Fluorescent lymphography-guided lymphadenectomy during robotic radical gastrectomy for gastric cancer. JAMA Surg. 154, 150-158 (2019). https://doi.org/10.1001/jamasurg.2018.4267

6. J. Liu, H.J. Li, Y.L. Luo, C.F. Xu, X.J. Du et al., Enhanced primary tumor penetration facilitates nanoparticle draining into lymph nodes after systemic injection for tumor metastasis inhibition. ACS Nano 13, 8648-8658 (2019). https://doi.org/ 10.1021/acsnano.9b03472

7. H. Cabral, J. Makino, Y. Matsumoto, P. Mi, H. Wu et al., Systemic targeting of lymph node metastasis through the blood vascular system by using size-controlled nanocarriers. ACS Nano 9, 4957-4967 (2015). https://doi.org/10.1021/nn507 0259

8. X.Y. Zhang, W.Y. Lu, Recent advances in lymphatic targeted drug delivery system for tumor metastasis. Cancer Biol. Med. 11, 247-254 (2014).

9. Z. Yan, F. Wang, Z. Wen, C. Zhan, L. Feng et al., LyP-1-conjugated PEGylated liposomes: a carrier system for targeted therapy of lymphatic metastatic tumor. J. Control. Release 157, 118-125 (2012). https://doi.org/10.1016/j.jconrel.2011. 07.034

10. X. Li, Q. Dong, Z. Yan, W. Lu, L. Feng et al., MPEG-DSPE polymeric micelle for translymphatic chemotherapy of lymph node metastasis. Int. J. Pharm. 487, 8-16 (2015). https://doi.org/10.1016/j.ijpharm.2015.03.074

11. W. Yu, R. Liu, Y. Zhou, H. Gao, Size-tunable strategies for a tumor targeted drug delivery system. ACS Cent. Sci. 6, 100-116 (2020). https://doi.org/10.1021/acscentsci.9b011 39

12. W. Zhang, F. Wang, C. Hu, Y. Zhou, H. Gao et al., The progress and perspective of nanoparticle-enabled tumor metastasis treatment. Acta Pharm. Sin. B 10, 2037-2053 (2020). https://doi.org/10.1016/j.apsb.2020.07.013

13. S.K. Golombek, J.N. May, B. Theek, L. Appold, N. Drude et al., Tumor targeting via EPR: Strategies to enhance patient responses. Adv. Drug Deliv. Rev. 130, 17-38 (2018). https:// doi.org/10.1016/j.addr.2018.07.007

14. J. Ding, J. Chen, L. Gao, Z. Jiang, Y. Zhang et al., Engineered nanomedicines with enhanced tumor penetration. Nano Today 29, 100800 (2019). https://doi.org/10.1016/j.nantod.2019. 100800

15. J. Chen, J. Ding, Y. Wang, J. Cheng, S. Ji et al., Sequentially responsive shell-stacked nanoparticles for deep penetration into solid tumors. Adv. Mater. 29, 1701170 (2017). https:// doi.org/10.1002/adma.201701170

16. P. Mi, H. Cabral, K. Kataoka, Ligand-installed nanocarriers toward precision therapy. Adv. Mater. 32, 1902604 (2020). https://doi.org/10.1002/adma.201902604 
17. S. He, Y. Jiang, J. Li, K. Pu, Semiconducting polycomplex nanoparticles for photothermal ferrotherapy of cancer. Angew. Chem. Int. Ed. 59, 10633-10638 (2020). https://doi.org/10. 1002/anie.202003004

18. J. Li, D. Cui, Y. Jiang, J. Huang, P. Cheng et al., Near-infrared photoactivatable semiconducting polymer nanoblockaders for metastasis-inhibited combination cancer therapy. Adv. Mater. 31, 1905091 (2019). https://doi.org/10.1002/adma.201905091

19. Y. Zhang, M. Jeon, L.J. Rich, H. Hong, J. Geng et al., Noninvasive multimodal functional imaging of the intestine with frozen micellar naphthalocyanines. Nat. Nanotechnol. 9, 631638 (2014). https://doi.org/10.1038/nnano.2014.130

20. Y. Zhang, D. Wang, S. Goel, B. Sun, U. Chitgupi et al., Surfactant-stripped frozen pheophytin micelles for multimodal gut imaging. Adv. Mater. 28, 8524-8530 (2016). https://doi. org/10.1002/adma.201602373

21. U. Chitgupi, N. Nyayapathi, J. Kim, D. Wang, B. Sun et al., Surfactant-stripped micelles for NIR-II photoacoustic imaging through $12 \mathrm{~cm}$ of breast tissue and whole human breasts. Adv. Mater. 31, 1902279 (2019). https://doi.org/10.1002/adma. 201902279

22. A. Beji, D. Horst, J. Engel, T. Kirchner, A. Ullrich, Toward the prognostic significance and therapeutic potential of HER3 receptor tyrosine kinase in human colon cancer. Clin. Cancer Res. 18, 956-968 (2012). https://doi.org/10.1158/1078-0432. CCR-11-1186

23. H. Xu, Y. Yu, D. Marciniak, A.K. Rishi, F.H. Sarkar et al., Epidermal growth factor receptor (EGFR)-related protein inhibits multiple members of the EGFR family in colon and breast cancer cells. Mol. Cancer Ther. 4, 435-442 (2005). https:// doi.org/10.1158/1535-7163.MCT-04-0280

24. H.-J. Liu, X. Luan, H.-Y. Feng, X. Dong, S.-C. Yang et al., Integrated combination treatment using a "smart" chemotherapy and microrna delivery system improves outcomes in an orthotopic colorectal cancer model. Adv. Funct. Mater. 28, 1801118 (2018). https://doi.org/10.1002/adfm.201801118

25. W. Zhang, K. Gilstrap, L. Wu, C.R. K, M.A. Moss et al., Synthesis and characterization of thermally responsive Pluronic F127-chitosan nanocapsules for controlled release and intracellular delivery of small molecules. ACS Nano 4, 6747-6759 (2010). https://doi.org/10.1021/nn101617n

26. H. Yang, Q.V. Le, G. Shim, Y.K. Oh, Y.K. Shin, Molecular engineering of antibodies for site-specific conjugation to lipid polydopamine hybrid nanoparticles. Acta Pharm. Sin. B 10, 2212-2226 (2020). https://doi.org/10.1016/j.apsb.2020.07.006

27. Y. Jiang, X. Zhao, J. Huang, J. Li, P.K. Upputuri et al., Transformable hybrid semiconducting polymer nanozyme for second near-infrared photothermal ferrotherapy. Nat. Commun. 11, 1857 (2020). https://doi.org/10.1038/s41467-020-15730-x

28. M. Cui, S. Liu, B. Song, D. Guo, J. Wang et al., Fluorescent silicon nanorods-based nanotheranostic agents for multimodal imaging-guided photothermal therapy. Nano-Micro Lett. 11, 73 (2019). https://doi.org/10.1007/s40820-019-0306-9

29. D. Wang, H. Wu, J. Zhou, P. Xu, C. Wang et al., In situ onepot synthesis of MOF-polydopamine hybrid nanogels with enhanced photothermal effect for targeted cancer therapy.
Adv. Sci. 5, 1800287 (2018). https://doi.org/10.1002/advs. 201800287

30. Z.-J. Chen, S.-C. Yang, X.-L. Liu, Y. Gao, X. Dong et al., Nanobowl-supported liposomes improve drug loading and delivery. Nano Lett. 20, 4177-4187 (2020). https://doi.org/ 10.1021/acs.nanolett.0c00495

31. W. Shan, R. Chen, Q. Zhang, J. Zhao, B. Chen et al., Improved stable indocyanine green (ICG)-mediated cancer optotheranostics with naturalized hepatitis B core particles. Adv. Mater. 30, 1707567 (2018). https://doi.org/10.1002/ adma.201707567

32. H.J. Li, J.Z. Du, X.J. Du, C.F. Xu, C.Y. Sun et al., Stimuliresponsive clustered nanoparticles for improved tumor penetration and therapeutic efficacy. Proc. Natl. Acad. Sci. U.S.A. 113, 4164-4169 (2016). https://doi.org/10.1073/pnas.15220 80113

33. A. Pittobarry, N.P.E. Barry, Pluronic $®$ block-copolymers in medicine: from chemical and biological versatility to rationalisation and clinical advances. Polym. Chem. 5, 3291-3297 (2014). https://doi.org/10.1039/C4PY00039K

34. D.H. Yu, Q. Lu, J. Xie, C. Fang, H.Z. Chen, Peptide-conjugated biodegradable nanoparticles as a carrier to target paclitaxel to tumor neovasculature. Biomaterials 31, 2278-2292 (2010). https://doi.org/10.1016/j.biomaterials.2009.11.047

35. H. Wang, G.R. Williams, J. Wu, J. Wu, S. Niu et al., Pluronic F127-based micelles for tumor-targeted bufalin delivery. Int. J. Pharm. 559, 289-298 (2019). https://doi.org/10.1016/j.ijpha rm.2019.01.049

36. Y. Zhang, W. Song, J. Geng, U. Chitgupi, H. Unsal et al., Therapeutic surfactant-stripped frozen micelles. Nat. Commun. 7, 11649 (2016). https://doi.org/10.1038/ncomms11649

37. Y. Zhang, L. Feng, J. Wang, D. Tao, C. Liang et al., Surfactantstripped micelles of near infrared dye and paclitaxel for photoacoustic imaging guided photothermal-chemotherapy. Small 14, 1802991 (2018). https://doi.org/10.1002/smll.201802991

38. J. Li, K. Pu, Semiconducting polymer nanomaterials as nearinfrared photoactivatable protherapeutics for cancer. Acc. Chem. Res. 53, 752-762 (2020). https://doi.org/10.1021/acs. accounts.9b00569

39. C. Xu, K. Pu, Second near-infrared photothermal materials for combinational nanotheranostics. Chem. Soc. Rev. 50, 1111-1137 (2021). https://doi.org/10.1039/D0CS00664E

40. D.Y. Oh, Y.J. Bang, HER2-targeted therapies - a role beyond breast cancer. Nat. Rev. Clin. Oncol. 17, 33-48 (2020). https:// doi.org/10.1038/s41571-019-0268-3

41. F. Meric-Bernstam, H. Hurwitz, K.P.S. Raghav, R.R. McWilliams, M. Fakih et al., Pertuzumab plus trastuzumab for HER2-amplified metastatic colorectal cancer (MyPathway): an updated report from a multicentre, open-label, phase 2a, multiple basket study. Lancet Oncol. 20, 518-530 (2019). https://doi.org/10.1016/S1470-2045(18)30904-5

42. A. Sartore-Bianchi, S. Marsoni, S. Siena, Human epidermal growth factor receptor 2 as a molecular biomarker for metastatic colorectal cancer. JAMA Oncol. 4, 19-20 (2018). https:// doi.org/10.1001/jamaoncol.2017.3323 
43. S. Togami, R. Kubo, T. Kawamura, S. Yanazume, M. Kamio, H. Kobayashi, Risk factors for lymphatic complications following lymphadenectomy in patients with endometrial cancer. Taiwan. J. Obstet. Gynecol. 59, 420-424 (2020). https://doi. org/10.1016/j.tjog.2020.03.015
44. D. Luo, S. Goel, H.J. Liu, K.A. Carter, D. Jiang et al., Intrabilayer ${ }^{64} \mathrm{Cu}$ labeling of photoactivatable, doxorubicin-loaded stealth liposomes. ACS Nano 11, 12482-12491 (2017). https:// doi.org/10.1021/acsnano.7b06578 